combination of genetics, multiple sera for defining antigens, and the difficulty of being sure of the specificity of the sera has produced a terminological jungle. Even the basic genetic map of the HLA region in man $^{3}$ does not impress the reader with the logical sequence, since the letters of the loci read in order D, B, C, and A. A further complication is that serological typing of the $\mathrm{D}$ region is performed on purified $\mathrm{B}$ lymphocytes as opposed to T cells and is sometimes known as $\mathrm{B}$ cell typing, easily confused with typing for the B locus. Even HLA does not stand for human leucocyte antigens, as one might have expected, but for the human leucocyte $A$, the A being the first system studied. So throughout the Bulletin the other antigens are defined HLA-A, $-\mathrm{B},-\mathrm{C}$, and -D.

If the reader is prepared to persevere with this terminology then the review articles are excellent, including descriptions of both serological and cellular typing. There is a full account of the matching of HLA and transplantation, and the relevance of different diseases to HLA types. Some complement components are coded for the HLA region and this is discussed by Lachmann and Hobart. ${ }^{4}$ The diseases related to HLA range from ankylosing spondylitis, liver disease, and Hodgkin's disease to leukaemia. In the final paper on the evolution and function of the HLA system Bodmer and Bodmer ${ }^{5}$ point out that "the HLA system contains a remarkable cluster of genes controlling cell surface determinants and immune functions, which between them generate an extraordinary amount of genetic variability in the human population. More than 300000000 genetically different individuals can be formed by the known alleles of the HLA/A, B, C, and D loci, and more than 30000000 of these have distinguishable combinations of antigens."

The HLA system has been extremely important in research in human genetics. Any further help expected from tissue typing in transplantation may, however, be limited, since the definition of additional antigens makes the chance of obtaining a good match most unlikely, even with the most efficient methods of co-operation and transport of organs. The main advances to be expected in organ transplantation with cadaver donors will come from better immunosuppressive drugs having a safer therapeutic index.

${ }^{1}$ Morris, P, Batchelor, J R, and Festenstein, H, British Medical Bulletin, 1978, 34, 259.

2 British Medical Bulletin, 1978, 34, 213. Published by the British Council at $£ 5$ and obtainable from 65 Davies Street, London W1Y 2AA.

${ }^{3}$ Bodmer, J G, British Medical Bulletin, 1978, 34, 233.

${ }^{4}$ Lachmann, P J, and Hobart, M J, British Medical Bulletin, 1978, 34, 247.

Bodmer, W F, and Bodmer, J G, British Medical Bulletin, 1978, 34, 309.

\section{Phantom limb pain}

The great French military surgeon Ambroise Paré is credited with the first description, in 1551, of the phenomenon of phantom limb. ${ }^{1}$ After amputation of a limb or some other part of the body such as a breast or the external genitals, the missing part may seem to the individual concerned to be still present and to have all the spatial characteristics of the real limb. This sensation has long been general knowledge-as shown by the carpenter in Moby Dick, ${ }^{2}$ who, on hearing Captain Ahab's story, stated that "a dismasted man never entirely loses the feeling of his old spar, but it will still be pricking him at times." The presence of a phantom limb is reported almost universally after amputation, ${ }^{3}$ but phantom limb pain is rare, most authorities ${ }^{3}{ }^{4}$ giving an incidence of
$3-5 \%$. The exact incidence of such pain is difficult to establish because of lack of agreement about whether the term includes transient aches and paraesthesiae or should be restricted to unbearable and permanent pain.

Phantom limb pain has to be clearly differentiated from local pains such as causalgia, pain in the stump, or pain secondary to a disc lesion. Typically it is dull, boring, and aching but it may occasionally be stabbing or shooting and similar to causalgia. Sunderland ${ }^{5}$ has defined several of its characteristics: its intermittent or continuous nature, its precipitation by peripheral stimulation or psychological stress, and its variable duration from seconds to days. Some patients have pain-free intervals lasting several years. Most get better, but some become permanently incapacitated.

The cause of phantom limb pain is unknown. Some writers have explained the whole matter in psychoanalytical terms, ${ }^{6}$ while others have implicated disordered mechanisms in the central nervous system. ${ }^{3}$ $\mathrm{A}$ central factor clearly plays a part in pathogenesis: the phantom limb possesses spatial qualities that are dependent on the parietal cortex. Cerebral lesions in the hemisphere contralateral to the pain may relieve it. ${ }^{8}$ One suggested mechanism is based on the theory that there are reverberating circuits within the central somatosensory system: when the output of these self-sustaining neurone pools exceeds a certain level, pain occurs. ${ }^{7}$ The circuits may be modified by afferent input, which may explain the relief of pain by local anaesthetic block ${ }^{9}$ or electrical stimulation. ${ }^{10}$ Henderson and Smyth" argued that if "the phantom does not result from persistent irritation of nerve ends it follows that we must look for the cause in the isolation of central mechanisms from their natural peripheral connection." They further concluded that several factors may be concerned, "for its manifestations may be derived from three levels in the nervous system (psychogenic, sensorimotor cortex and peripheral nerve)."

The alternative explanation is that peripheral mechanisms may predominate - a view supported by the finding that relief may occur after sympathetic block ${ }^{11}$ and by the absence of phantom limb pain in paraplegic patients with complete cord transection (though the pain has been found when the lesion is incomplete ${ }^{12}$ ). Further evidence implicating a peripheral mechanism has recently been deduced from the case of a 62-year-old man who had phantom limb pain after amputation of his left arm and, seven years later, developed recurrence of the pain when he had a herpes zoster infection affecting the left T2 dermatome, ${ }^{13}$-so providing "indirect evidence of the importance of primary afferents and their immediate connections in phantom limb pain."

Not only do we understand the pathogenic mechanisms of phantom limb pain poorly, but our treatment is also unsatisfactory. Despite many claims we still have no remedy. Treatment cannot be rational so long as aetiology remains obscure. Among methods that have been claimed to be successful are hypnosis, ${ }^{14}$ psychotherapy, ${ }^{15}$ mechanical percussion of the stump, ${ }^{16}$ local anaesthetic injection of the stump, ${ }^{17}$ section of sympathetic nerve, ${ }^{11}$ and a variety of leucotomies. ${ }^{18}$ Analgesics usually have no effect and, because of the risk of addiction, should be avoided. Tricyclic antidepressants and rarely carbamazepine have been found helpful. ${ }^{19}$ We urgently need further research into the pathogenesis of this crippling disorder.

\footnotetext{
1 Keynes, G, ed, The Apologie and Treatise of Ambroise Paré. Chicago, University of Chicago Press, 1952.

2 Melville, H, Moby Dick. New York, Dell, 1959

${ }^{3}$ Henderson, W R, and Smyth, G E, fournal of Neurology, Neurosurgery and Psychiatry, 1948, 11, 88.
} 
4 Solonen, K A, Acta Orthopaedica Scandinavica, 1962, Suppl No 54.

5 Sunderland, S, Nerves and Nerve Injuries. Baltimore, Williams and Wilkins, 1968.

${ }^{6}$ Ewalt, J R, Randall, G C, and Morris, H, Psychosomatic Medicine, 1947, 9, 118.

${ }^{7}$ Melzack, R, Advances in Neurology, 1974, 4, 319

${ }^{8}$ Head, H, and Holmes, G, Brain, 1911, 34, 102.

${ }^{9}$ Moore, D C, Regional Block: A Handbook for Use in the Clinical Practice of Medicine and Surgery, 4th edn. Springfield, Illinois, Charles C Thomas, 1965

${ }^{10}$ Shealy, C N, Advances in Neurology, 1974, 4, 775.

11 Gillis, L, British fournal of Surgery, 1964, 51, 87.

${ }^{12}$ Ruch, T C, Advances in Neurology, 1974, 4, 329.

${ }_{13}$ Wilson, P R, et al, Mayo Clinic Proceedings, 1978, 53, 336

${ }^{14}$ Cedercreutz, C, Acta Chirurgica Scandinavica, 1954, 107, 158

${ }^{15}$ Frazier, S H, Diseases of the Nervous System, 1966, 27, 441.

${ }^{16}$ Russell, W R, British Medical fournal, 1949, 1, 1024.

${ }_{17}$ Livingston, W K, Archives of Surgery, 1938, 37, 353.

18 Wilson, D H, and Chang, A E, Confinia Neurologica, 1974, 36, 61.

${ }^{19}$ Elliott, F, Little, A, and Milbrandt, W, New England fournal of Medicine, 1976, 295, 678 .

\section{Lymphocyte capping and carrier detection in Duchenne muscular dystrophy}

The most severe form of human muscular dystrophy, the Duchenne type, affects young boys and is known to be inherited by an X-linked recessive mechanism (as is the more benign and much less common Becker variety). Within the last 15 to 20 years many efforts have been made to attempt to identify women carriers of the genes responsible for these two conditions. If the Lyon hypothesis is accepted, then in the carrier female the X-chromosome carrying the Duchenne gene would be inactivated in about half of muscle nuclei, which would then be normal, while in the remaining half the normal chromosome would be inactivated, so that these nuclei would express the manifestations of the dystrophic gene. Some few women carriers do show minimal clinical evidence of muscular weakness (manifesting carriers), but this feature is not normally detectable unless precise quantitative methods of assessment of muscle power are used.

In patients with Duchenne type muscular dystrophy, the serum creatine kinase (CK) activity is appreciably raised, especially in the early stages, and in 1959 Ebashi et al ${ }^{1}$ showed that the CK activity is raised in the serum of some female carriers. Subsequently this test has been recognised as the most effective means of identifying carriers, though only between $50 \%$ and $80 \%$ of definite carriers (who have an affected male child or sib and another affected male relative) show raised CK activity. In 1969 Emery $^{2}$ suggested that Bayesian probability methods be used to calculate a risk factor based primarily on the actual level of $\mathrm{CK}$ activity in the putative carrier and her mother. This technique has been widely used, and recent evidence suggests that an active carrier detection programme combined with genetic counselling has led to a reduction in the births of affected boys, though information is still being collected. Additional impetus has been given to carrier detection programmes since it became possible to sex the fetus by amniocentesis and then to recommend therapeutic abortion of males. More recently still, the introduction of fetoscopy combined with fetal blood sampling has raised the possibility that it may be feasible to recommend abortion only of presumptively affected males. ${ }^{3}$

Detection of carriers is especially important in the sisters of dystrophic boys, since if the mother of the affected boy carries the gene then each of her daughters has a $50 \%$ chance of being a carrier. Nevertheless, many patients with Duchenne muscular dystrophy are isolated cases, having been born into families with no history of the disease. These examples are presumed to result from mutation occurring either in the mother or in the maternal grandmother. If such a mutation occurred in only some of the mother's ovarian cells then her daughters would be unlikely to be carriers. In 1935 Haldane $^{4}$ estimated that if the incidence of a lethal X-linked disorder remains in equilibrium in a population then one-third of all cases must result from genetic mutation, and in 1970 Gardner-Medwin, 5 following others, calculated that the mutation rate of this gene must be one of the highest recorded in human genetics. It has been estimated ${ }^{6}$ to lie between 4.6 and $9.4 \times 10^{-5}$.

Much work has recently been done to try to improve methods of carrier detection using techniques such as quantitative electromyography, muscle biopsy, ${ }^{78}$ and measurement of the incorporation of amino-acids into muscle polyribosomes in vitro. ${ }^{9}$ These methods have been tried as ways of identifying carriers in whom serum CK activity is normal.

More recent reports have suggested, firstly, that techniques based on the study of red blood cell membranes or of the white cells may be helpful, and, secondly, that the mutation rate in this condition may not be as high as has been thought. The abnormal release of cytoplasmic enzymes from the muscle cell into the serum seems to depend on changes in muscle membrane permeability. ${ }^{1011}$ Electron microscopic studies have shown that in patients with Duchenne dystrophy and in carriers there are also alterations in the surface membrane of the erythrocytes, and the biochemical behaviour of red cell membranes in blood samples obtained from patients with this disease and from carriers has been shown to be abnormal in several respects. ${ }^{12} 13$

These changes, as well as alterations in the shape of the erythrocytes, ${ }^{14}$ seem to be associated with altered membrane fluidity ${ }^{15} 16$ as shown by techniques such as electron spin resonance. Pickard et $a l^{17}$ have shown that these changes in membrane fluidity in B lymphocytes may influence the aggregation of fluorescent antigen-antibody complexes on their surface (the capping phenomenon). They found that in 61 patients with Duchenne, Becker, limb-girdle, facioscapulohumeral, and congenital muscular dystrophy there were fewer fluorescent lymphocytes that reached the capping stage, even when the total B lymphocyte count was normal. Capping was, however, normal in patients with myotonic dystrophy, motor neurone disease, polymyositis, and Kugelberg-Welander spinal muscular atrophy. In addition, just as Roses et al ${ }^{18}$ found biochemical abnormalities not only in erythrocyte ghosts in known female carriers of the gene but also in an unexpectedly high proportion of mothers of isolated cases, Pickard et al ${ }^{17}$ found a reduction in lymphocyte capping not only in known carriers but also in all but four mothers of isolated cases.

At first sight these findings, like those of Roses et al, , $^{18}$ suggest that many mothers of isolated cases-in whose sons the disease would be presumed to result from mutation-are in fact carriers of the gene. If confirmed, this finding must imply a mechanism such as selective fertilisation or a mutation rate several times higher in males than in females. The practical importance of these observations is obvious in relation to genetic counselling.

The findings reported by Pickard et $a l^{17}$ are important and deserve early replication. We must express caution, however, since many reports have failed to confirm all of the abnormalities in red cells reported in patients and carriers by Roses and his colleagues. ${ }^{18}$ Furthermore, there is evidence to indicate 